\title{
Optical Properties of Bis(histidinato)cobalt(III) Complexes
}

\author{
SVEN BAGGER and HANS PETER JENSEN
}

Chemistry Department A, Building 207, The Technical University of Denmark, DK-2800 Lyngby, Denmark

Dedicated to Jannik Bjerrum on the occasion of his 70th birthday

The isomer all-cis-[Co(L-his)(D-his) $]^{+}$has been resolved. Improved procedures for the preparation and separation of trans-imidazole- and transamine- $\left[\mathrm{Co}(\mathrm{L}-\mathrm{his})_{2}\right]^{+}$are given. The electronic spectra of all the geometrical isomers of bis(histidinato)cobalt(III) are discussed in terms of the angular overlap model.
An octahedral bis(histidinato)cobalt(III) complex gives rise to five geometrical isomers, four of which are chiral. The nine different theoretical structures are shown in Fig. 1.

The absorption spectra of the isomers trans-i, trans-a, trans-c and all-cis are reported in the literature. ${ }^{1,2}$ The circular dichroism (CD) spectra

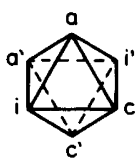

LL-trans-i

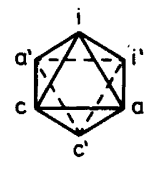

LL-trans-a
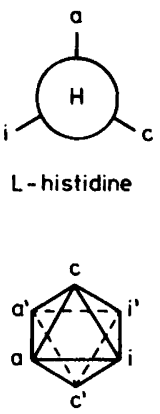

LL-trans-c

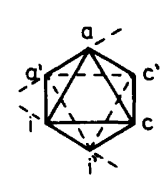

LD- $\wedge$-atl-cis
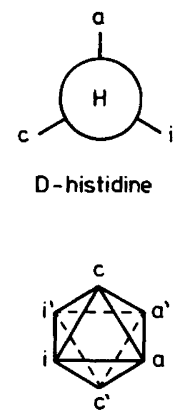

DD-trans-c

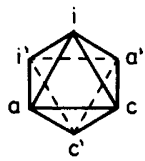

DD-trans-a

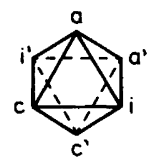

DD-trans-i

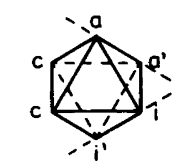

$D L-\Delta$-all-cis

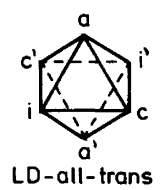

Fig. 1. Newman projections of histidine, and the structures of the bis(histidinato)cobalt(III) isomers. 
<smiles>NC(Cc1c[nH]cn1)C(=O)[O-]</smiles>

Fig. 2. Histidinate; L-histidine has the (S)-configuration in the Cahn-Ingold-Prelog notation.

of trans-i, trans-a and trans-c are also known. ${ }^{2}$ The achiral all-trans isomer, however, has not yet been isolated and characterized.

In this work CD spectrum of the all-cis isomer is reported.

Schmidtke ${ }^{3}$ has successfully used the angular overlap model (AOM), proposed by Schäffer and Jørgensen, ${ }^{4}$ to explain the absorption spectra of the three isomers known at that time, namely trans-i, trans-a and trans-c. We have reexamined the application of the model to bis(histidinato)cobalt(III) complexes taking into account all the spectral data now available.

\section{NOMENCLATURE}

In formulas histidinate (Fig. 2) is abbreviated his $^{-}$.

The letters $a, i$ and $c$ are used as symbols for amino, imidazole and carboxylate, respectively.

The IUPAC rules for the designation of absolute configurations of octahedral complexes ${ }^{5}$ may be adapted to all-cis-[Co(his) $\left.{ }_{2}\right]^{+}$using the pairs of nonorthogonal skew lines indicated in Fig. 1.

\section{EXPERIMENTAL RESULTS}

The racemic isomer all-cis-[Co(L-his)(D-his) $] \mathrm{Br}$ has been resolved chromatographically using a

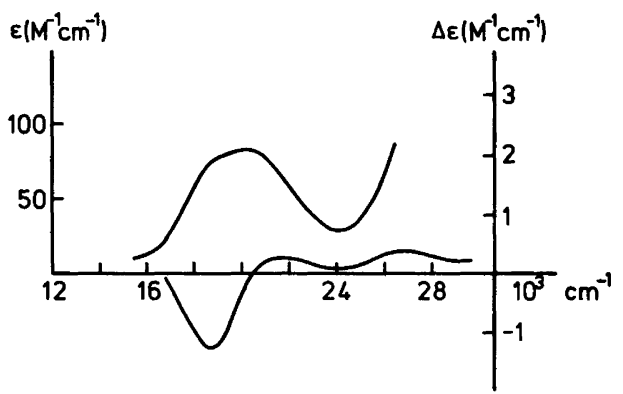

Fig. 3. Spectra of the $(-)_{589}$ antipode of the all-cis$[\mathrm{Co}(\mathrm{L}-\mathrm{his})(\mathrm{D}-\mathrm{his})]^{+}$isomer in $\mathrm{H}_{2} \mathrm{O}$.
Sephadex column. The antipodes separate into two distinct zones, indicating complete resolution.

The absorption and CD spectra are shown in Fig. 3.

An improved procedure for obtaining pure samples of trans-i-[Co(L-his $\left.)_{2}\right] \mathrm{ClO}_{4} \cdot 1 \frac{1}{2} \mathrm{H}_{2} \mathrm{O}$ and trans-a- $\left[\mathrm{Co}(\mathrm{L}-\mathrm{his})_{2}\right] \mathrm{ClO}_{4} \cdot 2 \mathrm{H}_{2} \mathrm{O}$ has been worked out. Previously the two isomers were separated by chromatography, ${ }^{6}$ whereas the new method makes use of the different crystallization rates of the perchlorates.

Resolution of rac-all-cis-[Co(D-his)(L-his) $] B r$. The complex is prepared as the bromide ${ }^{2}$ and is subsequently resolved chromatographically on SPSephadex C-25 with $0.1 \mathrm{M}$ aqueous sodium antimonyl-d-tartrate as eluent. Our column was 30 $\mathrm{cm}$ long and had a diameter of $3 \mathrm{~cm} ; 20 \mathrm{mg}$ complex was eluted with a flow-rate of $0.5 \mathrm{ml} / \mathrm{min}$. After $21 \mathrm{~h}$ two separate $1.5 \mathrm{~cm}$ broad zones had developed with centers 23 and $27 \mathrm{~cm}$ from the top of the column. The $(+)_{589}$ antipode is eluted first.

Preparation of trans-imidazole- $\left[\mathrm{Co}(\mathrm{L}-\mathrm{his})_{2}\right] \mathrm{ClO}_{4}$.$1 \frac{1}{2} \mathrm{H}_{2} \mathrm{O}$ (red isomer) and trans-amine- $\left[\mathrm{Co}(\mathrm{L}-h i s)_{2}\right]$ $\mathrm{ClO}_{4} .2 \mathrm{H}_{2} \mathrm{O}$ (orange isomer). $3.57 \mathrm{~g} \mathrm{CoCl}_{2} \cdot 6 \mathrm{H}_{2} \mathrm{O}$ $(15 \mathrm{mmol})$ and $4.65 \mathrm{~g} \mathrm{t}$-histidine $(30 \mathrm{mmol})$ are dissolved in $50 \mathrm{ml} \mathrm{H}_{2} \mathrm{O}$, and $0.5 \mathrm{~g}$ activated charcoal is added. The solution is heated to $75{ }^{\circ} \mathrm{C}$ and aerated for $90 \mathrm{~min}$. Then $1 \mathrm{ml} 30 \% \mathrm{H}_{2} \mathrm{O}_{2}$ is added, and the solution is kept at $75^{\circ} \mathrm{C}$ for $30 \mathrm{~min}$. The reaction mixture is cooled to $c a .0^{\circ} \mathrm{C}$, and the wall of the flask is scratched with a glass rod to insure that possible small amounts of unwanted products are precipitated. Subsequently the cooled solution is filtered. The volume is adjusted to $50 \mathrm{ml}$ with water, and a solution of $20 \mathrm{~g} \mathrm{NaClO} \mathrm{Na}_{4} \cdot \mathrm{H}_{2} \mathrm{O}$ in $50 \mathrm{ml} \mathrm{H}_{2} \mathrm{O}$ is added. After cooling in an ice bath for $30 \mathrm{~min}$ a precipitate of the red isomer is filtered off. Recrystallization from hot $\left(70{ }^{\circ} \mathrm{C}\right)$ water. The mother liquid from the precipitation of the red isomer is left in a refrigerator for a couple of days and crystals of the orange isomer slowly form. Recrystallization from hot $\left(70^{\circ} \mathrm{C}\right)$ water.

Apparatus. A Cary 11 spectrophotometer and a Roussel-Jouan Dichrograph II were used for the measurements of optical spectra.

\section{THEORETICAL RESULTS}

The semi-empirical angular overlap model ${ }^{4}$ is a useful tool for calculating energy levels in transition metal complexes. Application of the concept holohedrized symmetry, ${ }^{4}$ which for low-symmetry molecules is higher than the actual symmetry, is a simplifying approximation which makes the model easy to deal with. The literature ${ }^{3,4}$ should be consulted 
for a description of the model and its underlying principles.

The holohedrized symmetry of all the bis(histidinato)cobalt(III) isomers is $D_{2 h}$. On lowering the symmetry from $O_{h}$ to $D_{2 h}$ the ${ }^{1} T_{1 g} \leftarrow{ }^{1} A_{1 g}$ parent transition in cobalt(III) is decomposed into $B_{1 g} \leftarrow A_{1 g}, B_{2 g} \leftarrow A_{1 g}$, and $B_{3 g} \leftarrow A_{1 g}$.

In his treatment of the trans-c, trans-a, and trans-i isomers Schmidtke ${ }^{3}$ gave the energies of the three component transitions, abbreviated $\Delta E\left(\mathrm{~B}_{1}\right), \Delta E\left(\mathrm{~B}_{2}\right)$, and $\Delta E\left(B_{3}\right)$, in terms of the special $A O M$ parameters. We prefer to express these energies as functions of the usual cubic ligand field parameters for carboxylate $\left(\Delta_{c}\right)$, amine $\left(\Delta_{\mathrm{a}}\right)$, and imidazole $\left(\Delta_{\mathrm{i}}\right)$. This is possible for low-spin $d^{6}$-systems, as already pointed out by Schäffer and Jørgensen. ${ }^{4}$ Otherwise we have used the procedure described by Schmidtke, ${ }^{3}$ and we arrive at the following energy expressions:

trans-c:

$$
\begin{aligned}
& \Delta E\left(\mathrm{~B}_{1}\right)=\frac{1}{2}\left(\Delta_{\mathrm{a}}+\Delta_{\mathrm{i}}\right) \\
& \Delta E\left(\mathrm{~B}_{2}\right)=\Delta E\left(\mathrm{~B}_{3}\right)=\frac{1}{4}\left(\Delta_{\mathrm{a}}+\Delta_{\mathrm{i}}+2 \Delta_{\mathrm{c}}\right)
\end{aligned}
$$

trans-a:

$\Delta E\left(\mathrm{~B}_{1}\right)=\Delta E\left(\mathrm{~B}_{3}\right)=\frac{1}{4}\left(2 \Delta_{\mathrm{a}}+\Delta_{\mathrm{i}}+\Delta\right)$

$\Delta E\left(B_{2}\right)=\frac{1}{2}\left(\Delta_{i}+\Delta_{c}\right)$

trans-i:

$$
\begin{aligned}
& \Delta E\left(\mathrm{~B}_{1}\right)=\Delta E\left(\mathrm{~B}_{2}\right)=\frac{1}{4}\left(\Delta_{\mathrm{a}}+2 \Delta_{\mathrm{i}}+\Delta_{\mathrm{c}}\right) \\
& \Delta E\left(\mathrm{~B}_{3}\right)=\frac{1}{2}\left(\Delta_{\mathrm{a}}+\Delta_{\mathrm{c}}\right)
\end{aligned}
$$

all-cis:

$$
\begin{aligned}
& \Delta E\left(\mathrm{~B}_{1}\right)=\frac{1}{4}\left(2 \Delta_{\mathrm{a}}+\Delta_{\mathrm{i}}+\Delta_{\mathrm{c}}\right) \\
& \Delta E\left(\mathrm{~B}_{2}\right)=\frac{1}{4}\left(\Delta_{\mathrm{a}}+2 \Delta_{\mathrm{i}}+\Delta_{\mathrm{c}}\right) \\
& \Delta E\left(\mathrm{~B}_{3}\right)=\frac{1}{4}\left(\Delta_{\mathrm{a}}+\Delta_{\mathrm{i}}+2 \Delta_{\mathrm{c}}\right)
\end{aligned}
$$

$$
\text { all-trans: }
$$

$$
\begin{aligned}
& \dot{\Delta E}\left(\mathrm{~B}_{1}\right)=\frac{1}{2}\left(\Delta_{\mathrm{a}}+\Delta_{\mathrm{i}}\right) \\
& \Delta E\left(\mathrm{~B}_{2}\right)=\frac{1}{2}\left(\Delta_{\mathrm{i}}+\Delta_{\mathrm{c}}\right) \\
& \Delta E\left(B_{3}\right)=\frac{1}{2}\left(\Delta_{\mathrm{a}}+\Delta_{\mathrm{c}}\right)
\end{aligned}
$$

Acta Chem. Scand. A 32 (1978) No. 7
These equations immediately tell that all-cis and all-trans have three bands under the $T_{1 g} \leftarrow A_{1 g}$ parent transition, whereas each of the isomers trans-c, trans-a, and trans-i because of degeneracy only have two.

Using the assumption of Schmidtke ${ }^{3}$

$\Delta_{\mathrm{a}}>\Delta_{\mathrm{i}}>\Delta_{\mathrm{c}}$

which is in accordance with the spectrochemical series, ${ }^{7,8}$ it is easily deduced that the relative magnitudes of the transition energies are:

trans-c: $\quad \Delta E\left(\mathrm{~B}_{1}\right)>\Delta E\left(\mathrm{~B}_{2}\right)=\Delta E\left(\mathrm{~B}_{3}\right)$

trans-a: $\quad \Delta E\left(\mathrm{~B}_{1}\right)=\Delta E\left(\mathrm{~B}_{3}\right)>\Delta E\left(\mathrm{~B}_{2}\right)$

trans-i: $\quad \Delta E\left(\mathrm{~B}_{1}\right)=\Delta E\left(\mathrm{~B}_{2}\right)>\Delta E\left(\mathrm{~B}_{3}\right)$

all-cis: $\quad \Delta E\left(\mathrm{~B}_{1}\right)>\Delta E\left(\mathrm{~B}_{2}\right)>\Delta E\left(\mathrm{~B}_{3}\right)$

all-trans: $\Delta E\left(\mathrm{~B}_{1}\right)>\Delta E\left(\mathrm{~B}_{3}\right)>\Delta E\left(\mathrm{~B}_{2}\right)$

These five relations may be used for the assignment of the transitions in the experimental spectra.

\section{DISCUSSION}

It appears from eqns. (1) - (5) that if the energies of any three transitions in the five isomers are empirically known, the AOM will predict the energies of all the others.

Three of the transitions may be assigned and quite accurately read from the known experimental absorption spectra, namely the low-energy band of the trans-c isomer, and the high-energy bands of the trans-a and trans-i isomers, respectively. These three bands are marked by arrows in Fig. 4.

Simple calculations yield the values for the three cubic ligand field parameters as well as the transition energies listed in Table 1.

Table 1. Wavenumbers (in units of $10^{3} \cdot \mathrm{cm}^{-1}$ ) of the $T_{1 g} \leftarrow A_{1 g}$ components in [Co(his) $\left.)_{2}\right]^{+}$isomers.

\begin{tabular}{llll}
\hline Isomer & $\Delta E\left(\mathrm{~B}_{1}\right)$ & $\Delta E\left(\mathrm{~B}_{2}\right)$ & $\Delta E\left(\mathrm{~B}_{3}\right)$ \\
\hline trans-c & 21.9 & $18.7^{a}$ & $18.7^{a}$ \\
trans-a & $20.6^{a}$ & 18.1 & $20.6^{a}$ \\
trans-i & $20.0^{a}$ & $20.0^{a}$ & 19.3 \\
all-cis & 20.6 & 20.0 & 18.7 \\
all-trans & 21.9 & 18.1 & 19.3 \\
\hline
\end{tabular}

${ }^{a}$ Experimentally determined values. 

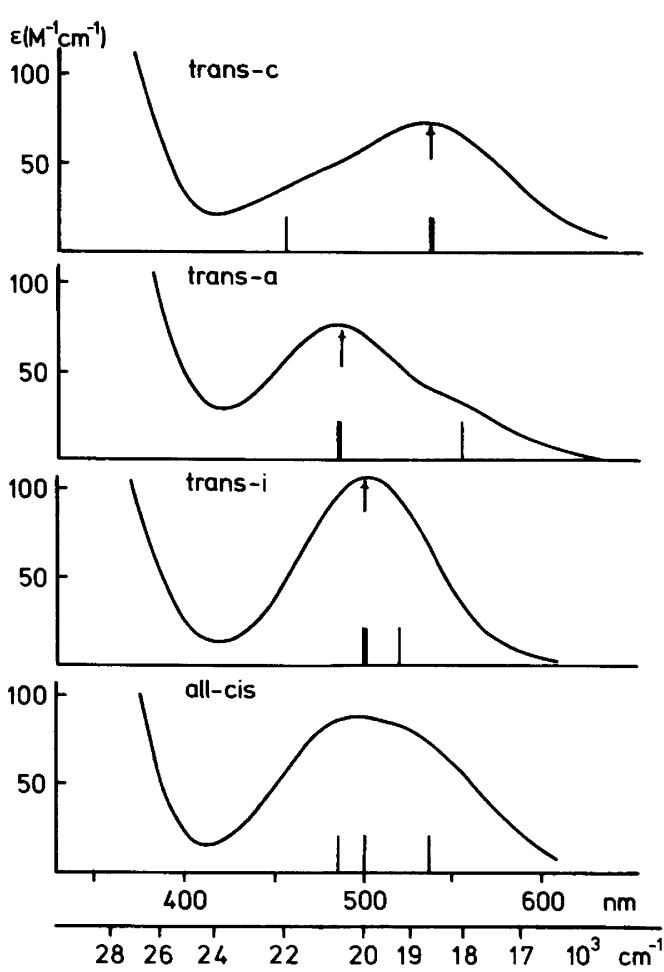

Fig. 4. Experimental absorption spectra of the four known geometrical isomers of the bis(histidinato)cobalt(III) complex. Vertical lines indicate the position of transitions from Table 1 .

The energy values from Table 1 are depicted as vertical lines in Fig. 4. The agreement between the experimental spectra and the AOM results is seen to be very satisfactory.

Table 1 includes a prediction of the unknown spectrum of the all-trans isomer, which so far has escaped isolation. ${ }^{2}$

The cubic ligand field parameters obtained, $\Delta_{\mathrm{a}}=23.1 \times 10^{3} \mathrm{~cm}^{-1}, \Delta_{\mathrm{i}}=20.7 \times 10^{3} \mathrm{~cm}^{-1}$ and $\Delta_{c}=15.5 \times 10^{3} \mathrm{~cm}^{-1}$, are in good agreement with expected values from the literature. ${ }^{7}$

The fact that all-cis-[Co(D-his)(L-his) $]^{+}$has been resolved into optical antipodes is a confirmation of its structure ${ }^{2,9}$ since the other possibility, all-trans$[\mathrm{Co}(\mathrm{D}-\mathrm{his})(\mathrm{L}-\mathrm{his})]^{+}$, has a symmetry centrum and would therefore be nonresolvable.

The CD spectra of the four chiral isomers of $\left[\mathrm{Co}(\mathrm{his})_{2}\right]^{+}$turn out to be of little use for the purpose of determining transition energies. Their shapes are typical for so-called "residual wing ab- sorptions", which arise when positive and negative contributions partially cancel each other. ${ }^{10}$

Since chirality vanishes on holohedrization the simple AOM used above is not appropriate for a detailed discussion of circular dichroism.

Acknowledgement. We wish to acknowledge the skilful technical assistance of Mrs. Lise Penzien.

\section{REFERENCES}

1. Zompa, L. J. Chem. Commun. (1969) 783.

2. Bagger, S., Gibson, K. and Sørensen, C. S. Acta Chem. Scand. 26 (1972) 2503.

3. Schmidtke, H. H. Chem. Phys. Lett. 4 (1969) 451.

4. a. Schäffer, C. E. and Jørgensen, C. K. K. Dan. Vidensk. Selsk., Mat.-Fys. Medd. 34 (1965), No. 13; b. Mol. Phys. 9 (1965) 401.

5. IUPAC Inorg. Chem. 9 (1970) 1.

6. Bagger, S. and Gibson, K. Acta Chem. Scand. 27 (1973) 3227.

7. Jørgensen, C. K. Modern Aspects of Ligand Field Theory, North-Holland, Amsterdam 1971, pp. $346-348$.

8. Epstein, L. M., Straub, D. K. and Maricondi, C. Inorg. Chem. 6 (1967) 1720.

9. Thorup, N. Acta Chem. Scand. A 31 (1977) 203.

10. Jensen, H. P. and Galsbøl, F. Inorg. Chem. 16 (1977) 1294. 